Conference Proceedings Paper

\title{
Microbiome Analysis of Healthy and Bleached Freshwater Sponges
}

\author{
Itskovich V. ${ }^{1}$, Kaluzhnaya O. ${ }^{1}$, Prathiviraj R. ${ }^{2}$, G. Seghal Kiran ${ }^{2}$, Selvin $\mathrm{J}^{2}$. \\ 1 Limnological Institute, Siberian Branch of the Russian Academy of Sciences, 3, Ulan-Batorskaya, Irkutsk, \\ Russia, 664033 \\ 2 Department of Microbiology, School of Life Sciences, Pondicherry University, Puducherry, 605014, India \\ * Correspondence: itskovich@mail.ru; Tel.: +7-914-883-56-41
}

\begin{abstract}
:
Sponge microbiome is an indicator of both natural and antropogenic stresses. In aquatic ecology, metagenomic approaches makes it possible to investigate complex microbial communities and their interactions within the host as well as with environment. A metagenomic study was performed among 4 different samples of healthy and diseased freshwater sponges belong to Lubomirskia baikalensis species collected from Baikal Lake, Bolshie Koty, Russia. The analysis of nucleotide composition of the $16 \mathrm{~S}$ rRNAmetagenomic libraries will be carried out on an IlluminaMySeq sequencer. The PCR amplification of the V3-V4 16S rRNA ribosomal gene was used in this study. The primary analysis of NGS sequencing data, removal of short and chimeric sequences, clustering in OTUs (operational taxonomic units), assessment of biodiversity by calculating ACE, Chao1, and Shannon indices will be carried out using the Mothur v.1.22.0 program (http://www. mothur.org). The Pyrosequencing pipeline program (http://pyro.cme.msu.edu) will be used to determine species diversity and taxonomic composition and compare communities. Data cluster analysis will be performed using the Complete Linkage Clustering program, which is part of the Pyrosequencing pipeline. A comparative analysis of the metagenomes of diseased and healthy sponges showed that diseased sponges undergo a variety of nonspecific changes in the bacterial composition, in particular, an increase in the number of Flavobacteria and a decrease in Alphaproteobacteria and Saprospirae. The composition of the bacterial community is probably influenced by the type of sponge, as well as the degree of disease affection. The results contribute to understanding the nature of changes in symbiotic relationships during the development of discoloration syndrome in freshwater sponges.
\end{abstract}

Keywords: Porifera; metagenome; sponges

\section{Introduction}

Sponges (Porifera) are the oldest and most primitive multicellular animals [1]. The importance of studying of the sponges is due to their basal position in the multicellular tree, combined with insufficient knowledge of their origin, evolution, and taxonomy. Sponges are also important as biofiltrators and bioindicators of the status of aquatic ecosystems and producers of biological active substances [2]. 
Sponges are ideal bioindicators of the environmental state, due to their simple body structure, species richness and filter-feeding life style[2,3,4,5]. Sponges pump large quantities of water and have ability to concentrate a wide range of chemicals from both the suspended and dissolved phases of the water [3,4]. Additionally, sponges are tolerant of some pollutants, making them suitable model organisms for monitoring studies [5,6].

Baikal sponges is an endemic family of 14 species [7,8]. Recently, Baikal sponges have been getting sick and dying off $[9,10,11]$. Baikal sponges are closely associated with bacteria, and the composition of the symbiotic community changes during the development of the disease [9,12]. We studied bacterial diversity by metagenomic analysis by sequencing Illumina $16 \mathrm{~S}$ rDNA libraries.

\section{Experiments}

Sequencing protocol

The analysis of nucleotide composition of the $16 \mathrm{~S}$ rRNA metagenomic libraries will be carried out on an Illumina MySeq sequencer. The PCR amplification of the V3-V4 16S rRNA ribosomal gene was used in this study. The primary analysis of NGS sequencing data, removal of short and chimeric sequences, clustering in OTUs (operational taxonomic units), assessment of biodiversity by calculating ACE, Chao1, and Shannon indices will be carried out using the Mothur v.1.22.0 program (http://www. mothur.org). The Pyrosequencing pipeline program (http://pyro.cme.msu.edu) will be used to determine species diversity and taxonomic composition and compare communities. Data cluster analysis will be performed using the Complete Linkage Clustering program, which is part of the Pyrosequencing pipeline.

In silico metagenomic analysis

The metagenomic analysis was performed using the MG-RAST server V 4.0.3 software package [13]. The MG-RAST analysis workflow allows users to perform genus-level identification forms in GLE (The graph module) reads; with access to a base called files for detailed investigations at the domain, order, family, and genus- level. The study was intended by performing a sequence similarity search of the 16s RNA gene against the available bacterial RNA gene sequence database. Best hits were classified based on the percentage of identifying and query coverage. The $16 \mathrm{~S}$ workflow will be useful in identifying pathogens in a mixed sample or understanding the composition of a microbial community. The phylogeny analysis of query sequence with the closely related sequence of blast results was performed followed by multiple sequence alignment. Based on the MG-RAST server we predict sequence diversity, disparity index, K-mer profile at the genus-level classification of the selected seven shrimp gut microbiome sample.

\section{Results and Discussion.}

A comparative analysis of sick and healthy sponges, as well as our results with those obtained earlier, showed that diseased sponges undergo a variety of nonspecific changes in the bacterial composition. Thus, when comparing healthy and diseased samples of L. baikalensis, similar changes in the symbiotic composition were found, which were expressed in a significant increase in the 
number of Flavobacteria and a decrease in Alphaproteobacteria and Saprospirae. However, other deseased sample of L. baikalensis has a significantly increased number of Bacteroidia. When comparing two other L. baikalensis specimens, an increase in Saprospirae is observed in a diseased sponge, as well as a decrease in Betaproteobacteria.

Thus, when comparing healthy and diseased samples, we did not reveal specific changes in the bacterial composition in diseased sponges. These results are consistent with those obtained earlier. Thus, when studying the diversity of $16 \mathrm{~S}$ rRNA genes in the microbial community of L. baikalensis with discolored tissue areas, the dominance of Cyanobacteria and a low number of representatives of Bacteroidetes and Betaproteobacteria were noted [9]. At the same time, a comparison of the microbiomes of healthy and diseased sponges collected in 2011 and 2015 showed an increase in the number of Bacteroidetes and Proteobacteria, as well as the absence of a specific pathogen [12].

\section{Conclusions.}

A comparative analysis of the metagenomes of diseased and healthy sponges showed that diseased sponges undergo a variety of nonspecific changes in the bacterial composition, in particular, an increase in the number of Flavobacteria and a decrease in Alphaproteobacteria and Saprospirae. The composition of the bacterial community is probably influenced by the type of sponge, as well as the degree of disease affection. The results contribute to understanding the nature of changes in symbiotic relationships during the development of discoloration syndrome in freshwater sponges.

Acknowledgments: The reported study was funded by RFBR and DST according to the research project № 19-54-45034, by RFBR according to the research project № 20-04-00868 and basic funding, project number 0279-2021-0011.

Author Contributions: Itskovich V., Kaluzhnaya O., and Selvin J. conceived and designed the experiments, wrote the paper; Prathiviraj R., G. and Seghal Kiran performed the experiments and analyzed the data, Itskovich $\mathrm{V}$. supervised research.

Conflicts of Interest: The authors declare no conflict of interest. The funding sponsors had no role in the design of the study; in the collection, analyses, or interpretation of data; in the writing of the manuscript, and in the decision to publish the results.

\section{Abbreviations}

The following abbreviations are used in this manuscript:

OTU: operational taxonomic unit

\section{References}

1. Bell J. J. 2008. The functional roles of marine sponges. Estuarine Coastal and Shelf Science 79: 341-352.https://doi.org/10.1007/s10750-013-1799-8

2. Bell J.J., Biggerstaff A., Bates T. et al.2017. Sponge monitoring: moving beyond diversity and abundance measures. EcologicalIndicators 78: 470-488. DOI: 10.1016/j.ecolind.2017.03.001 Cebrian E., UrizM.J., Turon X. et al. 2007. Sponges as biomonitors of heavy metals in spatial and temporal surveys in northwestern mediterranean: multispecies comparison. Environmental Toxicology and Chemistry 26(11): 2430-2439.DOI: 10.1897/07-292.1 
3. López-Legentil S., Song B., McMurray S.E. et al. 2008. Bleaching and stress in coral reef ecosystems: Hsp70 expression by the giant barrel sponge Xestospongia muta. Molecular Ecology 17: 1840-1850. doi: 10.1111/j.1365-294X.2008.03667.x.

4. Selvin J, Priya S.S., Kiran G.S. et al. 2009. Sponge associated marine bacteria as indicators of heavy metal pollution. Microbiological Research 164(3): 352-63.

5. Selvin J., Ninawe A.S., Kiran G.S. et al. 2010. Sponge-Microbial Interactions: Ecological Implications and Bioprospecting Avenues. Critical Reviews Microbiology 36: 82-90.

6. Efremova S., Itskovich V., Parfenova V. et al. 2002. Lake Baikal: a unique place to study evolution of sponges and their stress response in an environment nearly unimpaired by anthropogenic perturbation. Cellular and Molecular Biology48: 359 - 371.

7. Efremova S.M. 2004. New genus and new species of sponges from family LubomirskiidaeRezvoj, 1936. In Timoshkin, O.A. (ed), Index of animal species inhabiting lake Baikal and its catchment area, vol. 1. Lake Baikal, Book 2. Nauka, Novosibirsk: 1261-1278. (in Russian).

8. Efremova, S.M., 2001. Sponges (Porifera). In Timoshkin, O.A. (ed), Index of animal species inhabiting lake Baikal and its catchment area, vol. 1. Lake Baikal, Book 1. Nauka, Novosibirsk: 182-192. (in Russian).

9. Kaluzhnaya O.V., Itskovich V.B. 2015. Influence of Baikal sponge bleaching to taxonomic composition of symbiotic microorganisms. Russian Journal of Genetics 51 (11): 1-6. https://doi.org/10.1134/S1022795415110071

10. Timoshkin O.A., Samsonov D.P., Yamamuro M.et al. 2016.Rapid ecological change in the coastal zone of Lake Baikal (East Siberia): is the site ofthe world's greatest freshwater biodiversity in danger? Journal Great Lakes Research 42:487-497. DOI: 10.1016/j.jglr.2016.02.011

11. Itskovich V.B., Shigarova A.M., Glyzina O.Y. et al. 2018. Heat shock protein 70 (Hsp70) response to elevated temperatures in the endemic Baikal sponge Lubomirskiabaicalensis. Ecological Indicators 88:1-7. DOI: 10.1016/j.ecolind.2017.12.055

12. Belikov S., Belkova N., Butina T., Chernogor L., Martynova-Van Kley A., Nalian A., Rorex C., Khanaev I., Maikova O., Feranchuk S. Diversity and shifts of the bacterial community associated with Baikal sponge mass mortalities . PLoS ONE. 2019. Vol. 14, N 3. P. 1-19.

13. Meyer F, Paarmann D, D'Souza M, Olson R, Glass EM, Kubal M, Paczian T, Rodriguez A, Stevens R, Wilke A, Wilkening J, Edwards RA (2008) The metagenomics RAST server - a public resource for the automatic phylogenetic and functional analysis of metagenomes. BMC Bioinformatics. 9: 386. article distributed under the terms and conditions of the Creative Commons by Attribution (CC-BY) license (http://creativecommons.org/licenses/by/4.0/). 